\title{
One-dimensional free-electron laser equations without the slowly varying envelope approximation
}

\author{
C. Maroli* \\ INFN- Mi, Via Celoria, 1620133 Milano, Italy \\ V. Petrillo \\ Università degli Studi di Milano, INFN-Mi, Via Celoria,16 20133 Milano, Italy \\ M. Ferrario \\ INFN-LNF, Via E. Fermi, 4000044 Frascati, Rome, Italy \\ (Received 4 May 2011; published 18 July 2011)
}

\begin{abstract}
A set of one-dimensional equations has been deduced in the time domain from the Maxwell-Lorentz system with the aim of describing the free-electron laser radiation without using the slowly varying envelope approximation (SVEA). These equations are valid even in the case of arbitrarily short electron bunches and of current distributions with ripples on the scale of or shorter than the wavelength. Numerical examples are presented, showing that for long homogeneous bunches the new set of equations gives results in agreement with the SVEA free-electron laser theory and that the use of short or prebunched electron beams leads to a decrease of the emission lethargy. Furthermore, we demonstrate that in all cases in which the backward low frequency wave has negligible effects, these equations can be reduced to a form similar to the usual 1D SVEA equations but with a different definition of the bunching term.
\end{abstract}

DOI: 10.1103/PhysRevSTAB.14.070703

PACS numbers: 41.60.Cr

\section{INTRODUCTION}

The usual equations describing the free-electron laser radiation process were deduced in the framework of the slowly varying envelope approximation (SVEA) [1-6]. This procedure requires that all the characteristic lengths $L$ (for instance: the pulse modulation width, the length of the gradients, the dimension of the electron beam $L_{b}$, the gain length $L_{G}$, the cooperation length $L_{C}$ ) are much longer than the wavelength $\lambda$ of the radiation, $L \gg \lambda$. Under this hypothesis, (i) the second order wave equation can be simplified in a first order one, (ii) the regressive wave, resonant on a low frequency, is disregarded, and (iii) the shorter interval of length that can be resolved is just the wavelength $\lambda$. The SVEA equations, in the 1D version $[3,4,6]$ and in their 3D extension [5], are at the basis of the development of several numerical codes [7-13] extensively and successfully used in the projects and in the interpretation of almost all free-electron laser (FEL) experiments. Few works [14-17] reintroduced the backward wave in the model, associating to the particle equations not one, but a couple of radiation equations, written for two wave packets centered, respectively, on two different single resonances and correlated only via the electron dynamics. Another characteristic length, the wavelength

\footnotetext{
*Maroli@mi.infn.it

Published by the American Physical Society under the terms of the Creative Commons Attribution 3.0 License. Further distribution of this work must maintain attribution to the author $(s)$ and the published article's title, journal citation, and DOI.
}

of the second FEL resonance $\lambda_{b} \approx 2 \lambda_{w}$ ( $\lambda_{w}$ undulator period), is in this way introduced with the further much stringent assumption $L \gg \lambda_{b}$. The system of equations written in Ref. [14], however, does not constitute a tool suitable for studying the emission from short or prebunched beams, because, even if it represents an extension of the usual SVEA system, the radiation field is assumed to be the superposition of two pulses both structured with slowly varying amplitudes. Superradiance and coherent spontaneous emission produced in short bunches have been the object of the studies proposed in Refs. [18,19]. In the first paper [18], an approach in the frequency domain is developed, while a time-domain model is presented in the second one [19]. In both analyses, the radiation is assumed to have a broad bandwidth around the high frequency resonance, but the contribution of other parts of the spectrum is neglected together with the second derivatives in the radiation equation. More general theories and codes have been then developed where the beam equations do not contain any average on the undulator wavelength and the fields are broadband [12] and work is continuously in progress along this line [20]. In this paper we derive a set of 1D FEL equations from the Maxwell-Lorentz system without using the SVEA, which is valid for arbitrarily short electron bunches as well as for current longitudinal distributions with ripples on or under the scale of the wavelength. No hypotheses on the bandwidth are assumed and, therefore, the solution has components coming from all the spectral regions. We will refer to this model as the NOSVEA system of equations. Furthermore, we have developed a code solving these equations. Numerical 
examples are presented, showing that for long homogeneous bunches the NOSVEA equations give results in agreement with the usual SVEA FEL theory. We are able to show, on the basis of this self-consistent theory, that the radiation coming from short or prebunched electron beams is characterized by a lethargy time shorter than that of long bunches and that the SVEA model often underestimates the saturation power. Situations where the backward wave can play a role are presented. Finally, we demonstrate that, under the assumption that the backward low frequency wave is negligible, these new equations can be reduced to the usual SVEA 1D equations, apart from the fact that the bunching term is computed on an average length smaller than the wavelength, thereby retrieving a model very close to those of Refs. [19,21].

\section{THE MODEL EQUATION}

The following set of one-dimensional equations describing the dynamics of a beam of $N_{p}$ electrons in an helical undulator and the consequent FEL radiation can be deduced from the Maxwell-Lorentz system:

$$
\begin{aligned}
\frac{d z_{j}(t)}{d t} & =\frac{c}{\bar{\gamma}_{j}(t)} P_{j}(t) \\
\frac{d P_{j}(t)}{d t} & =-\frac{c a_{w 0}}{\sqrt{2} \gamma_{0}^{2}} \frac{1}{\bar{\gamma}_{j}(t)}\left[\frac{\partial}{\partial z}\left[A(z, t) e^{i k_{w} z}+\text { c.c. }\right]\right]_{z=z_{j}(t)} \\
\frac{\partial^{2} A}{\partial t^{2}}-c^{2} \frac{\partial^{2} A}{\partial z^{2}} & =-\frac{4 \pi e^{2} n_{s} a_{w 0}}{\sqrt{2} m \gamma_{0}} \sum_{j=1, N_{p}} \frac{e^{-i k_{w} z_{j}(t)}}{\bar{\gamma}_{j}(t)} \delta\left[z-z_{j}(t)\right] .
\end{aligned}
$$

In (1) $z$ is the longitudinal coordinate, $t$ is the time, $z_{j}(t)$ the instantaneous electron longitudinal coordinate, $P_{j}(t)=$ $\beta_{j \|}(t) \gamma_{j}(t) / \gamma_{0}$ the normalized momentum, and $\gamma_{j}(t)$ the Lorentz factor of the $j$ th electron. Furthermore, $\gamma_{0}$ is the average value of $\gamma_{j}(t=0), \beta_{j \|}(t)=v_{j \|}(t) / c$ is the normalized longitudinal electron velocity, $\bar{\gamma}_{j}(t)=\gamma_{j}(t) / \gamma_{0}$, $-e$, and $m$ are the electron charge and mass, respectively, $n_{s}$ is the superficial electron density, and $\delta$ is the Dirac distribution. The undulator vector potential is described by the expression

$$
\hat{A}_{w}(z)=\frac{a_{w 0}}{\sqrt{2}}\left(e^{-i k_{w} z} \hat{e}+\text { c.c. }\right)
$$

with $a_{w 0}$ the wiggler parameter, $k_{w}$ the wiggler wave number, and $\hat{e}=\frac{1}{\sqrt{2}}\left(\hat{e}_{x}+i \hat{e}_{y}\right)$. The radiation vector potential is represented by

$$
\hat{A}(z, t)=A(z, t) \hat{e}+\text { c.c. }
$$

Both $\hat{A}$ and $\hat{A}_{w}$ have been written in the Coulomb gauge and normalized with respect to $m c^{2} / e$. Only a few standard hypotheses have been done in deducing system (1), i.e., (i) we have supposed that expression (2) is valid everywhere, not only on the $z$ axis, (ii) we have disregarded space charge, (iii) we have assumed that the transverse components of the generalized electron momenta vanish, and (iv) that the radiation potential $|A(z, t)|$ is smaller than $a_{w 0}$ all along the radiation process. Then, we simply write the radiation as the sum of two generic terms:

$$
A(z, t)=A_{p}(z, t)+A_{r}(z, t) .
$$

The set of equations,

$$
\begin{aligned}
& \left(\frac{\partial}{\partial t}+c \frac{\partial}{\partial z}\right) A_{p}(z, t)=Z(z, t) \\
& \left(\frac{\partial}{\partial t}-c \frac{\partial}{\partial z}\right) A_{r}(z, t)=-Z(z, t),
\end{aligned}
$$

is completely equivalent to the last of Eqs. (1) if $Z(z, t)$ is the solution of the equation:

$$
\frac{\partial}{\partial z} Z(z, t)=-\frac{1}{2 c} S(z, t)
$$

where $S$ is the right-hand side of the radiation equation in (1), i.e.,

$$
S(z, t)=-\frac{4 \pi e^{2} n_{s} a_{w 0}}{\sqrt{2} m \gamma_{0}} \sum_{j=1, N_{p}} \frac{e^{-i k_{w} z_{j}(t)}}{\bar{\gamma}_{j}(t)} \delta\left[z-z_{j}(t)\right] .
$$

From the former definition of $S$ and solving (6) with the condition $\lim _{z \rightarrow-\infty} Z(z, t)=0$ for each $t \geq 0$, one obtains

$$
\begin{aligned}
Z(z, t) & =\frac{4 \pi e^{2} n_{s} a_{w 0}}{2 \sqrt{2} m c \gamma_{0}} \sum_{j=1, N_{p}} \frac{e^{-i k_{w} z_{j}(t)}}{\bar{\gamma}_{j}(t)} \int_{-\infty}^{z} d z^{\prime} \delta\left[z^{\prime}-z_{j}(t)\right] \\
& =\frac{4 \pi e^{2} n_{s} a_{w 0}}{2 \sqrt{2} m c \gamma_{0}} \sum_{j<z} \frac{e^{-i k_{w} z_{j}(t)}}{\bar{\gamma}_{j}(t)}
\end{aligned}
$$

where the last sum is performed over all the electrons of the beam behind the point $z$. Our equations therefore are

$$
\begin{gathered}
\left(\frac{\partial}{\partial t}+c \frac{\partial}{\partial z}\right) A_{p}(z, t)=\frac{4 \pi e^{2} n_{s} a_{w 0}}{2 \sqrt{2} m c \gamma_{0}} \sum_{j<z} \frac{e^{-i k_{w} z_{j}(t)}}{\bar{\gamma}_{j}(t)} \\
\left(\frac{\partial}{\partial t}-c \frac{\partial}{\partial z}\right) A_{r}(z, t)=-\frac{4 \pi e^{2} n_{s} a_{w 0}}{2 \sqrt{2} m c \gamma_{0}} \sum_{j<z} \frac{e^{-i k_{w} z_{j}(t)}}{\bar{\gamma}_{j}(t)} \\
\frac{d z_{j}(t)}{d t}=\frac{c}{\bar{\gamma}_{j}(t)} P_{j}(t) \\
\frac{d P_{j}(t)}{d t}=-\frac{c a_{w 0}}{\sqrt{2} \gamma_{0}^{2}} \frac{1}{\bar{\gamma}_{j}(t)}\left[\frac{\partial}{\partial z}\left[\left(A_{p}+A_{r}\right) e^{i k_{w} z}+\text { c.c. }\right]\right]_{z=z_{j}}
\end{gathered}
$$

Equations (9)-(12) are equivalent to the system (1), but have the advantage that the equations for the radiation are of first order, so that one can use the same numerical integration schemes of the SVEA system. Furthermore, they do not contain delta functions in the source terms, permitting their numerical calculation without the necessity of spatial averages. The emitted power $P$ for unit of surface $S$ can be written as 


$$
F=\frac{d P}{d S}=\frac{m^{2} c^{5}}{2 \pi e^{2}}\left[\left|\frac{\partial A_{p}}{\partial z}\right|^{2}-\left|\frac{\partial A_{r}}{\partial z}\right|^{2}\right] .
$$

In this equation, one can clearly distinguish a progressive and a regressive flux.

From the Maxwell-Lorentz system (1), one can also deduce the usual SVEA equation with a procedure that we shortly recall. Imposing that

$$
A(z, t)=M(z, t) e^{i(k z-c k t)}
$$

and supposing a slow variation of the amplitude $M(z, t)$, with consequent cancellation of the second derivatives, the following equation can be retrieved:

$$
\left(\frac{\partial}{\partial t}+c \frac{\partial}{\partial z}\right) M(z, t)=-i \frac{4 \pi e^{2} n_{s} a_{w 0}}{2 \sqrt{2} m c k \gamma_{0} L_{m}} \sum_{s} \frac{e^{-i \theta_{s}(t)}}{\bar{\gamma}_{s}(t)}
$$

with $k=k_{w} \beta_{0} /\left(1-\beta_{0}\right), \quad \beta_{0} \approx\left[1-\left(1+a_{w 0}^{2}\right) / \gamma_{0}^{2}\right]^{1 / 2}$ and where $\theta_{s}(t)=\left(k+k_{w}\right) z_{s}(t)-c k t$ are the phase angles of the electrons in the ponderomotive wave. The term on the right-hand side of Eq. (15) is proportional to the bunching factor $b(z, t)$ as is commonly defined in the framework of the SVEA, the sum over the integer $s$ being extended over all the electrons for which $z-L_{m} / 2<$ $z_{s}(t)<z+L_{m} / 2$, with the average length $L_{m}$ equal to the radiation wavelength $\lambda$.

\section{NUMERICAL RESULTS}

\section{A. NOSVEA solution in the case of high energy, long and moderately short electron beams}

Equations (9)-(12) have been integrated numerically starting from noise and the results compared with those obtained by using the SVEA model described by Eq. (15). Typical results are presented in Fig. 1 and in Fig. 2 for bunches long and moderately short with respect to the wavelength. The case presented in Fig. 1 describes the physics of a long bunch with $L_{b}=800 \mu \mathrm{m}(I=12 A)$, while the second one in Fig. 2 can be classified as a situation representative of moderately short bunches $\left(L_{b} \gtrsim \lambda\right)$ and has $L_{b}=20 \mu \mathrm{m}(I=480 A)$. In both figures the red curves give the average power $\langle P\rangle=\frac{1}{L_{\text {pulse }}} \int F d z d S$ vs $z / L_{G}\left(L_{\text {pulse }}\right.$ being the length of the radiation wave packet and $L_{G}$ the gain length), solutions of Eqs. (9)-(12), while the blue curves are the usual SVEA results, obtained by integration of Eq. (15), the computational steps being very much shorter in the NOSVEA case, because the spatial and temporal oscillations on the wavelength have to be resolved.

For long bunches, as in Fig. 1, there is an initial difference of 1 or 2 orders of magnitude in the power emitted, which is reported in detail in the window at the upper left corner of the figure. The oscillations visible in the NOSVEA signal over the first few gain lengths have the period $\lambda_{w}=2 \pi / k_{w}$ of the undulator and can be fully explained by a short-time analysis of the NOSVEA

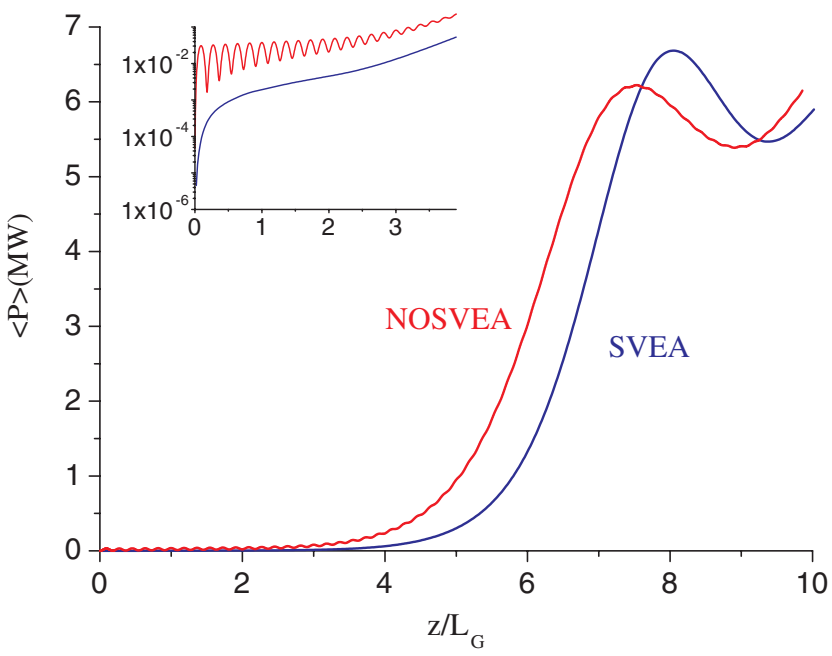

FIG. 1. Average power $\langle P\rangle$ vs $z / L_{G}$ in the case SVEA [solution of Eq. (15)] and NOSVEA [solution of Eqs. (9)-(12)] for the beam length $L_{b}=800 \mu \mathrm{m}$. The beam charge is $Q=$ $3.210^{-11} C, \gamma_{0}=100, a_{w 0}=1.47, \lambda_{w}=2.8 \mathrm{~cm}$, the beam radius $r_{b}=50 \mu \mathrm{m}$, and the resonant wavelength is $\lambda=4.42 \mu \mathrm{m}$. The current $I$ is flattop.

equations. After these few gain lengths, however, the power growth and the saturation values shown by the two curves in Fig. 1 become actually very similar with only small differences that remain within few percents and could be attributed to the different numerical schemes used for the integration of the two systems.

On the contrary, for shorter bunches as in Fig. 2, the details of the growth are also qualitatively different and the saturation power is larger in the NOSVEA case by a factor between 2 and 3. Another difference is that the lethargy presented by the radiation emitted by short bunches in the treatment without the SVEA is shorter and the radiation presents a very early growth characterized by

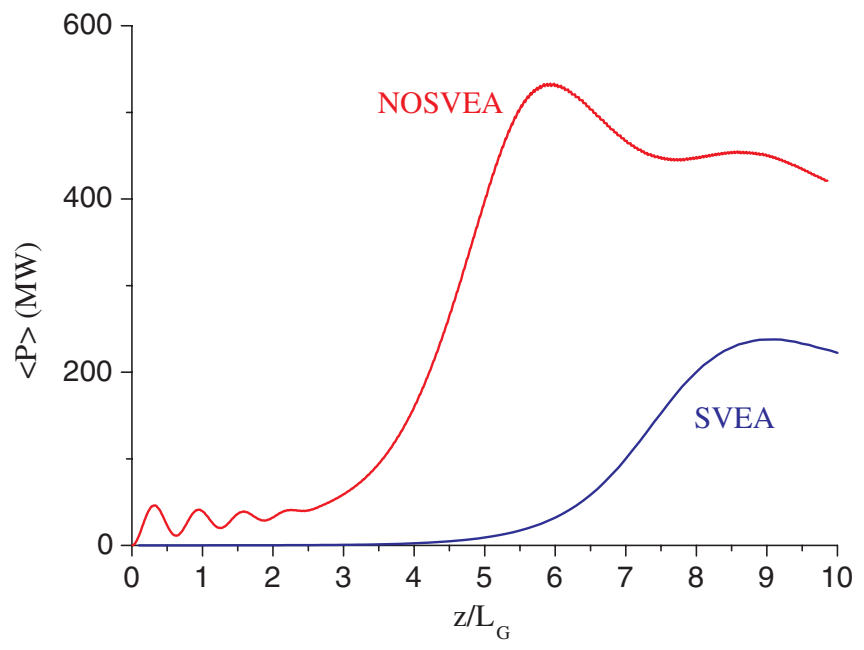

FIG. 2. Average power $\langle P\rangle$ vs $z / L_{G}$ in the case SVEA [solution of Eq. (15)] and NOSVEA [solution of Eqs. (9)-(12)] for the beam length $L_{b}=20 \mu \mathrm{m}$. The other parameters are the same as in Fig. 1. 
the same oscillations as before whose spatial periodicity is the wiggler wavelength $\lambda_{w}$. This large amount of spontaneous emission shown by short beams was first observed in Refs. [22,23], studied then in detail in [18,19,24,25] and connected with the presence of large gradients in the current profile. One could advance a possible alternative explanation of this enhanced spontaneous emission by the following reasoning. The exponential growth in the usual SVEA solution is due to the linear response of the system around the unstable equilibrium with $M=0, b=0$. Therefore, the SVEA source term of the radiation equation represented by the bunching factor $b$, which is evaluated by definition on a length exactly equal to the wavelength $\lambda$, at the beginning of the calculation is usually set equal to a very low value by accurately loading the electrons of the beam in the bucket (the quiet start). If instead the initial value of the bunching factor is large, an additional strong spontaneous emission appears due to the longitudinal acceleration of the electrons and the growth of the radiation is no more exponential, as, for instance, in situations of prebunched beams. In fact, in the NOSVEA model the source term in Eqs. (9) and (10) is a sum involving all the electrons behind the point $z$ and is not uniformly small, even if the electrons are quietly loaded. The resulting spontaneous emission is therefore always larger than in the SVEA case. When the beam is short, the initial difference in the power emitted is of several orders of magnitude, can be appreciated also in the linear graph of Fig. 2 and dominates the growth of the radiation along the whole process.

In Fig. 3 we have summarized the differences between SVEA and NOSVEA calculations as a function of the ratio between the beam length $L_{b}$ and the wavelength $\lambda$. In ordinate we report the difference between the SVEA $P_{\text {SVEA }}$ and the NOSVEA $P_{\text {NOSVEA }}$ first peak powers $\Delta_{1}=$ $P_{\text {NOSVEA }}-P_{\text {SVEA }}$ and the difference of the corresponding

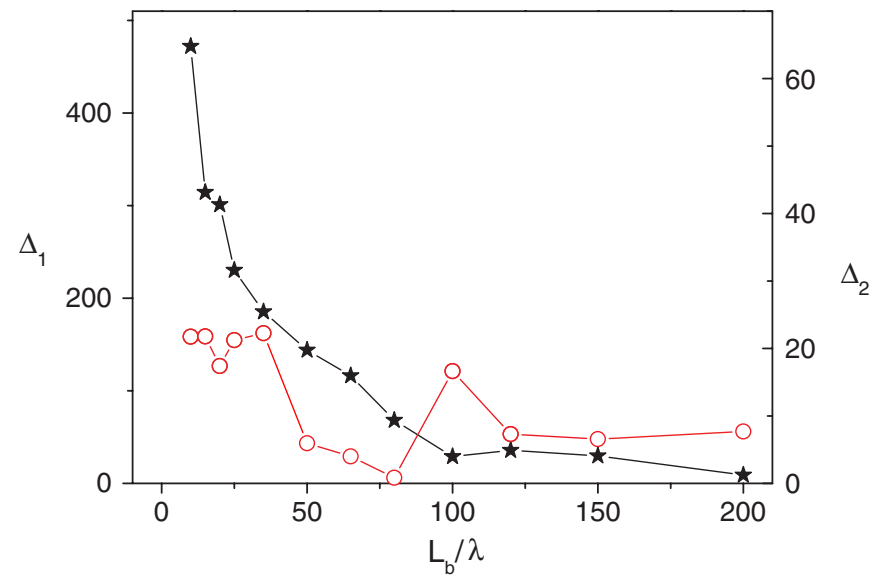

FIG. 3. Difference between the SVEA $P_{\text {SVEA }}$ and the NOSVEA $P_{\text {NOSVEA }}$ first peak powers $\Delta_{1}=P_{\text {NOSVEA }}-P_{\text {SVEA }}$ and difference of the corresponding saturation lengths $\Delta_{2}=$ $\left|L_{\text {NOSVEA }}-L_{\text {SVEA }}\right|$ vs $L_{b} / \lambda . \Delta_{1}$ (stars) is in MW, $\Delta_{2}$ (circles) in $\mathrm{cm}$. saturation lengths $\Delta_{2}=\left|L_{\text {NOSVEA }}-L_{\text {SVEA }}\right|$. Appreciable differences in both quantities occur only for roughly $L_{b} / \lambda<20$.

\section{B. NOSVEA solution in the case of high energy, prebunched and short electron beams}

In this paragraph and in the next one we present two examples of long wavelength radiation in the range of terahertz and microwaves. These situations, though less interesting from the point of view of applications, are useful to investigate the prebunched regime and the role of the backward wave $A_{r}(z, t)$. Situations in which the electron beam is much shorter than the wavelength can be found, for instance, in the field of the FEL terahertz production [26]. Figures 4 and 5 refer to a case in which $\lambda=254 \mu \mathrm{m}$ and $L_{b}=150 \mu \mathrm{m}$. In this case the beam is strongly prebunched and as one can see from the logarithmic plot in Fig. 4 the power growth is no more a simple exponential. In Fig. 5 snapshots of the power flux $F$ vs the coordinate $z$ along the electron beam are shown at a very early stage of the process and at four different positions inside the undulator. The black curve is the rough data, while the red one is the average over 100 spatial steps. A continuous release of backward wave whose wavelength is $\lambda_{b}=\lambda_{w} \frac{1+\beta_{0}}{\beta_{0}} \approx 2 \lambda_{w}$, behind the electron beam, can be observed. The slippage length $L_{s, b}$ of the backward wave is defined as the length of the region where the regressive wave propagates freely, i.e., $L_{s, b}=\left(1+\beta_{0}\right) c t \approx 2 z$. Because of the relatively large value of $\gamma_{0}$, this wave slips immediately out of the beam toward negative values of $z$ and does not interact deeply with the electrons, so the regressive power flux presents always a moderately low level. At later stages, the power on the beam becomes so large that the backward wave flux in comparison is negligible.

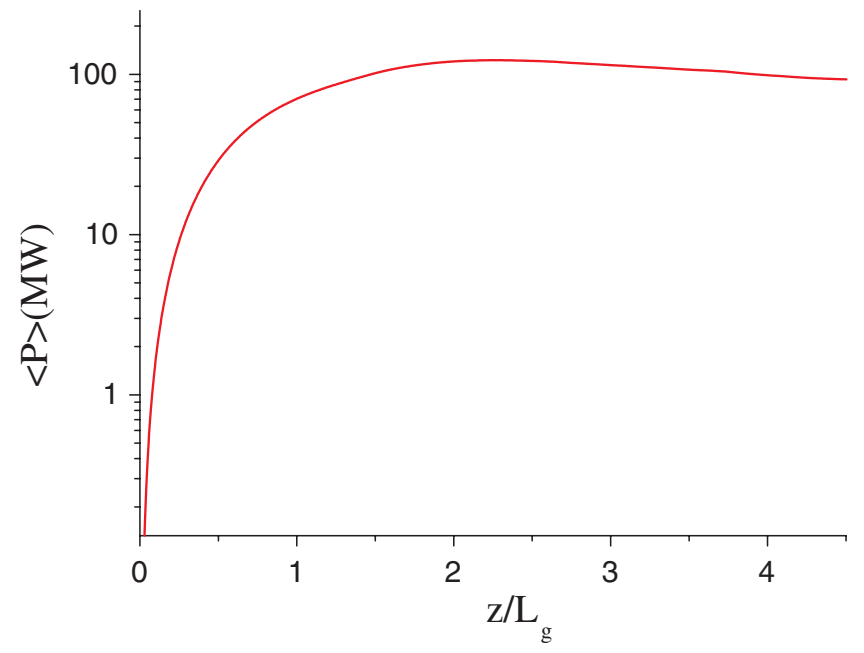

FIG. 4. Average power $\langle P\rangle$ vs $z / L_{g}$ in the case NOSVEA [solution of Eqs. (9)-(12)] for $\lambda=254 \mu \mathrm{m}, L_{b}=150 \mu \mathrm{m}$, $\lambda_{w}=8 \mathrm{~cm}, \gamma=23.5, Q=20 \mathrm{pC}$, and $a_{w 0}=1.583$. 

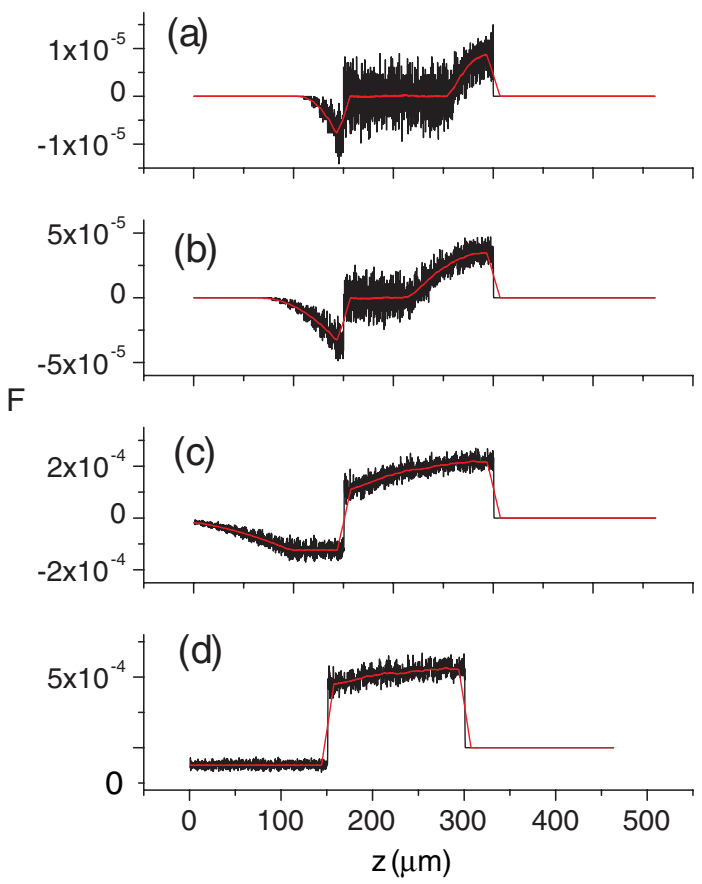

FIG. 5. Power flux $F=\frac{d P}{d S}$ vs the coordinate $z$ along the beam at (a) $\quad z / L_{G}=7.2 \times 10^{-3}, \quad$ (b) $z / L_{G}=1.43 \times 10^{-2}$, (c) $z / L_{G}=3.6 \times 10^{-2}$, and (d) $z / L_{G}=5.76 \times 10^{-2}$, for the following values of the parameters $\lambda=254 \mu \mathrm{m}, L_{b}=$ $150 \mu \mathrm{m}, \lambda_{w}=8 \mathrm{~cm}, \gamma_{0}=23.5, Q=20 \mathrm{pC}$, and $a_{w 0}=1.583$.

\section{NOSVEA solution in the case of low energy, prebunched and short electron beams}

The short-time analysis of the NOSVEA equations shows that the level of the backward wave scales as $1 / \gamma_{0}$.

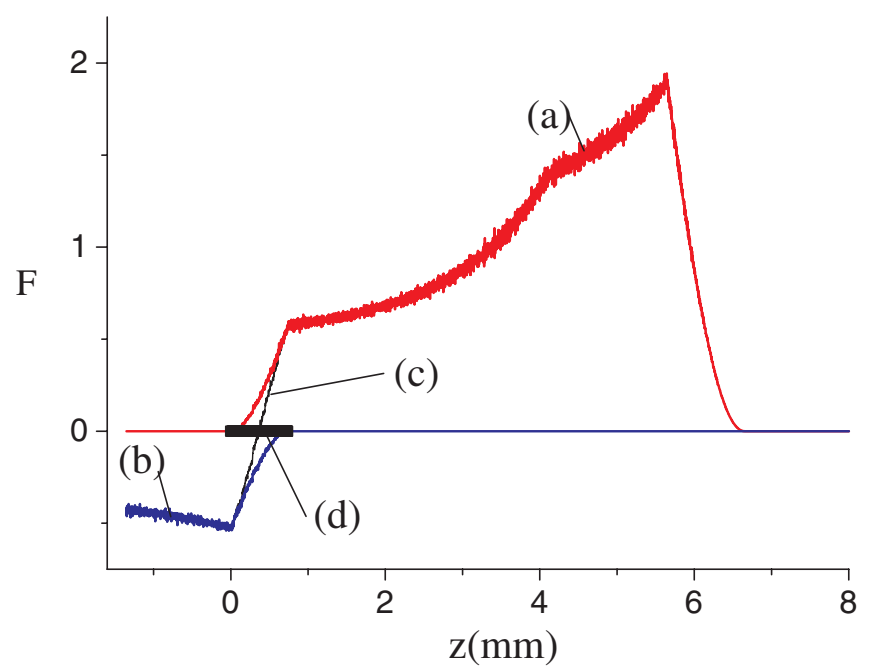

FIG. 6. Forward flux [red curve (a)], backward flux (truncated at the left of the figure) [blue curve (b)], total flux [black line (c)], and electron beam (d) vs the coordinate $z$ along the beam at the position $c t=3.3 L_{G}$ inside the undulator for $\gamma_{0}=1.155$, $L_{b}=1 \mathrm{~mm}, Q=32 \mathrm{pC}, \lambda_{w}=2 \mathrm{~cm}, a_{w 0}=1.47, \lambda=2 \mathrm{~cm}$, and $\lambda_{b}=6 \mathrm{~cm}$.
It follows that this wave is important when the Lorentz factor of the beam is small. In this condition, the slippage length of the forward wave $L_{s, f}=\left(1-\beta_{0}\right) c t=\lambda N_{u}$, with $N_{u}$ number of the undulator periods and $\lambda=\frac{\left(1-\beta_{0}\right)}{\beta_{0}} \lambda_{w}$, is of the same order as the slippage length of the backward wave $L_{s, b}=\lambda_{b w} N_{u}=\frac{1+\beta_{0}}{1-\beta_{0}} \lambda N_{u}$ and the growths of the two waves are comparable. Figure 6 shows a case with $\gamma_{0}=1.155$. The red curve (a) represents the forward flux at the position $c t=3.3 L_{G}$ along the undulator, the blue curve (b) is the backward flux (which is truncated at the left of the figure), the black line (c) is the total flux, and the black dots (d) give the position of the electron beam. In this case the backward flux is not much smaller than the forward flux, so that the negative total flux just behind the trailing edge of the beam becomes visible.

\section{NOSVEA FORMULATION WITHOUT BACKWARD WAVES}

In the preceding analysis we have shown that the situations where the backward wave is strong regard principally beams with weak energy leading to production of waves of low frequency. On the contrary, when the beam Lorentz factor is large, the backward wave is generally not important provided that it is not excited from the exterior and a NOSVEA formulation for the forward wave only is sufficient to cover the most interesting cases.

We have already shown that from the general equations (1) it is possible to deduce the SVEA equation (15) by admitting that the function $M(z, t)$ defined in (14) is a slowly varying function of both space and time. This condition of slow behavior is sufficient but not necessary for reducing Eq. (9) to (15). In fact, starting again by (9) and changing to the new variable,

$$
N_{p}(z, t)=-\frac{i}{k} \frac{\partial A_{p}(z, t)}{\partial z} e^{-i(k z-c k t)},
$$

we find without any restriction:

$$
\left(\frac{\partial}{\partial t}+c \frac{\partial}{\partial z}\right) N_{p}(z, t)=-i \frac{4 \pi e^{2} n_{s} a_{w 0}}{2 \sqrt{2} m c k \gamma_{0} L_{m}} \sum_{s} \frac{e^{-i \theta_{s}(t)}}{\bar{\gamma}_{s}(t)}
$$

which is valid in the only limit that the length $L_{m}$ used in the average process is sufficiently small to permit the identification of the average value $\left\langle N_{p}(z, t)\right\rangle$ with the actual value $N_{p}(z, t)$. With a similar procedure, but starting from Eq. (10), we can deduce a further equation similar to (17) which uses backward phases $\theta_{s}(t)=\left(k+k_{w}\right) z_{s}(t)+c k t$ for the quantity

$$
N_{r}(z, t)=-\frac{i}{k} \frac{\partial A_{r}(z, t)}{\partial z} e^{-i(k z+c k t)}
$$

that accounts for the contributions of the backward field.

By comparing Eqs. (17) and (15), we see that the two functions $N_{p}(z, t)$ and $M(z, t)$ satisfy the same equation, the 


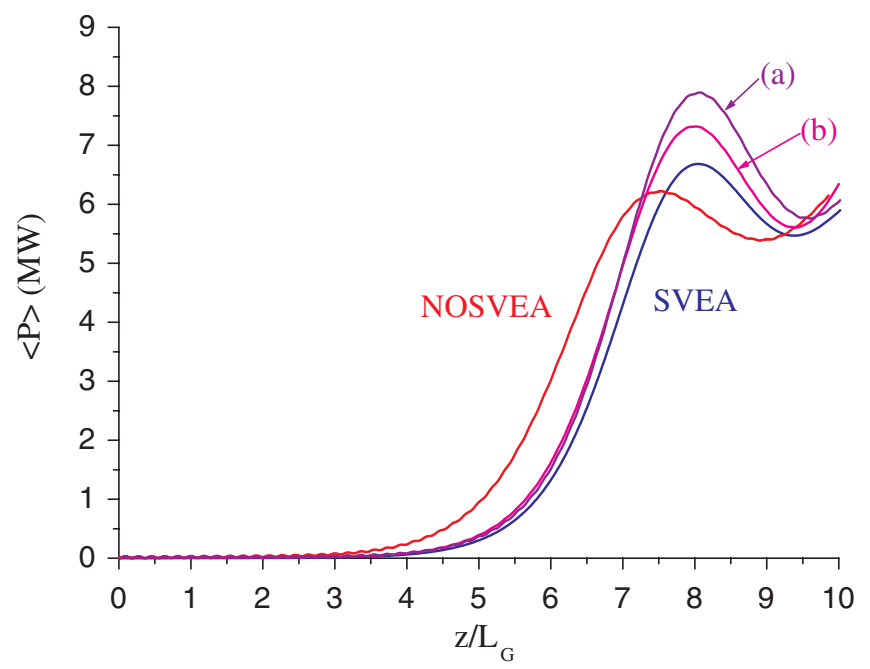

FIG. 7. Average power vs $z / L_{G}$ in the same case of Fig. 1 and solution of Eq. (17) with (a) $L_{m}=0.25 \lambda$ and (b) $L_{m}=0.75 \lambda$.

only difference regarding the choice of the average length $L_{m}$. In the usual SVEA procedure $L_{m}=\lambda$ but, in general, $L_{m}$ should be taken equal, in both equations, to the shortest characteristic length in the problem. Furthermore the two quantities $N_{p}(z, t)$ and $M(z, t)$ appear in the particle equations in the same way when the regressive field $A_{r}(z, t)$ is negligible and when $\frac{k_{w}}{k} \approx\left(1+a_{w 0}^{2}\right) /\left(2 \gamma_{0}^{2}\right) \ll 1$. It follows that $N_{p}(z, t)$ and $M(z, t)$ are equal at all times if they satisfy the same initial and boundary conditions. An equation similar to Eq. (17) has been proposed in Ref. [19]. We will refer to Eq. (17) as to the NOSVEA/no-backward model.

To support the preceding conclusion regarding the identity of $N_{p}(z, t)$ and $M(z, t)$, we give in the two following figures, 7 and 8, the solution of Eq. (17) for different decreasing values of the average length $L_{m}$ and using the

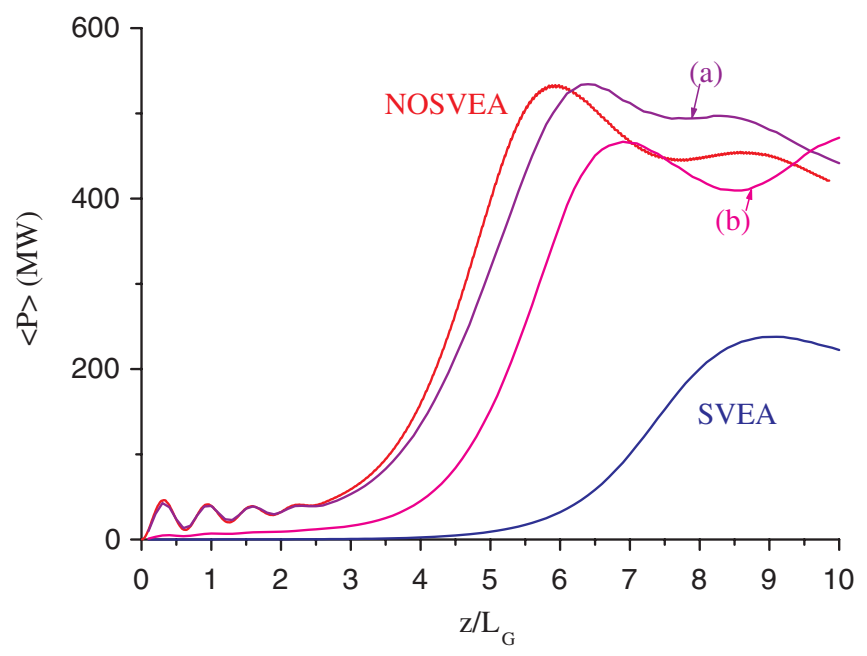

FIG. 8. Average power vs $z / L_{G}$ in the same cases of Fig. 2 and solution of Eq. (17) with (a) $L_{m}=0.1 \lambda$ and (b) $L_{m}=0.5 \lambda$. same parameters of Figs. 1 and 2, respectively, in which the backward wave is completely negligible.

In the case of long bunches, Fig. 7 shows that the codes SVEA, NOSVEA, and NOSVEA/no-backward all give very similar results. In the case of short bunches, Fig. 8 shows that the code based on Eq. (17) gives results that approach the NOSVEA results based on Eqs. (9)-(12) when the average length is progressively decreased and that the process tends to convergence when $L_{m}$ becomes shorter than the shortest characteristic length in the problem, as could also be seen by using the integral equation approach of Ref. [27]. As a direct consequence, in all cases where the regressive field is negligible and after a careful examination of the characteristic lengths involved, the code based on Eq. (17) can be used in place of that based on Eq. (9) with considerable saving of computer time.

\section{CONCLUSIONS}

We have written a set of equations valid outside the SVEA limits which can be used to investigate the FEL radiation when the electron bunch is short or presents density gradients on the wavelength scale. We have developed a numerical code for integrating these equations and the results have been compared with those given by the usual SVEA approach. We have also demonstrated that, if the backward wave is weak, another set of equations, already presented in the literature, can be directly derived from our equations. We have given numerical results where the three models (SVEA, NOSVEA, and NOSVEA/ no-backward) give very similar results, situations outside the SVEA limit where the NOSVEA model give results similar to the NOSVEA/no-backward model, and finally situations characterized by a strong regressive flux where it is necessary to use the complete NOSVEA model to have a full description of the radiation field. We have confirmed that short bunches present a strong initial spontaneous emission, shorter lethargy, and larger saturation values.

\section{ACKNOWLEDGMENTS}

The authors are deeply indebted to Dr. Luca Giannessi for his continuous interest and numerous enlightening discussions during this work.

[1] H. Haus, IEEE J. Quantum Electron. 17, 1427 (1981).

[2] G. Dattoli, A. Marino, A. Renieri, and F. Romanelli, IEEE J. Quantum Electron. 17, 1371 (1981).

[3] R. Bonifacio, C. Pellegrini, and L. Narducci, Opt. Commun. 50, 373 (1984).

[4] W. Colson, Phys. Lett. 59A, 187 (1976).

[5] E. Scharlemann, J. Appl. Phys. 58, 2154 (1985).

[6] W. Colson, Classical Free-Electron Laser Theory (NorthHolland Physics Elsevier, Netherlands, 1990), Chap. 5, p. 115. 
[7] G. Dattoli and P. L. Ottaviani, J. Appl. Phys. 86, 5331 (1999).

[8] E. Jong, W. Fawley, and E. Scharlemann, Proc. SPIE 1045, 18 (1994).

[9] S. Reiche, Nucl. Instrum. Methods Phys. Res., Sect. A 429, 243 (1999).

[10] L. Saldin, E. Schneidmiller, and M. V. Yurkov, Nucl. Instrum. Methods Phys. Res., Sect. A 429, 233 (1999).

[11] L. Giannessi, Phys. Rev. ST Accel. Beams 6, 114802 (2003).

[12] H. Freund, Phys. Rev. ST Accel. Beams 8, 110701 (2005).

[13] A. Bacci, M. Ferrario, C. Maroli, V. Petrillo, and L. Serafini, Phys. Rev. ST Accel. Beams 9, 060704 (2006).

[14] N. Piovella, V. Petrillo, C. Maroli, and R. Bonifacio, Phys. Rev. Lett. 72, 88 (1994).

[15] V. Petrillo and C. Maroli, Phys. Rev. E 62, 8612 (2000).

[16] C. Maroli, Opt. Commun. 208, 155 (2002).

[17] C. Maroli and V. Petrillo, Opt. Commun. 183, 139 (2000).

[18] N. Piovella, Phys. Plasmas 6, 3358 (1999).

[19] B. McNeil, G. Robb, and D. A. Jaroszinski, Opt. Commun. 165, 65 (1999).
[20] L. Campbell and B. McNeil, in Proceedings of the 32nd International Free-Electron Laser Conference (FEL 2010), Malmo, Sweden (Max-Lab Stockholm, Uppsala FEL Centre), MPOB30.

[21] L. Campbell, R. Martin, and B. McNeil, in Proceedings of the 31st International Free Electron Laser Conference (FEL 09), Liverpool, UK (STFC Daresbury Laboratory, Warrington, 2009), MPOC39.

[22] F. Ciocci et al., Phys. Rev. Lett. 70, 928 (1993).

[23] D. A. Jaroszinski et al., Phys. Rev. Lett. 71, 3798 (1993).

[24] G. Dattoli et al., J. Opt. Soc. Am. 10, 2136 (1993).

[25] G. Dattoli et al., Nucl. Instrum. Methods Phys. Res., Sect. A 393, 339 (1997).

[26] H. Hama and M. Yasuda, in Proceedings of the 31st International Free Electron Laser Conference (FEL 09), Liverpool, UK (Ref. [21]), TUPC69.

[27] F. Ciocci et al., Insertion Devices for Synchrotron Radiation and Free-Electron Lasers (World Scientific, Singapore, 2000). 